\title{
Joint Associations of Aerobic-Based Physical Activity and Muscle-Strengthening Activities on Metabolic Syndrome
}

\author{
Jungjun Lim¹, Soyoung Park ${ }^{1}$, Joon-Sik Kim ${ }^{1}$ \\ ${ }^{1}$ Department of Physical Education, College of Education, Seoul National University, Seoul \\ 08826, Korea \\ Correspondence: Joon-Sik Kim \\ Department of Physical Education, College of Education, Seoul National University, Seoul \\ 08826, Korea \\ E-mail:joonsik@snu.ac.kr
}

Running title: Combined physical activity and metabolic syndrome 


\section{Joint Associations of Aerobic-Based Physical Activity and}

\section{Muscle-Strengthening Activities on Metabolic Syndrome}

4 Abstract

5 OBJECTIVES: The study aims to examine whether meeting the combined guidelines of

6 accelerometer-assessed moderate to vigorous physical activity (MVPA) and self-reported

7 muscle-strengthening activity (MSA) simultaneously are associated with reduced odds of

8 metabolic syndrome (MetS) than meeting neither or one of the guidelines among the

9 Koreans.

METHODS: This cross-sectional analysis included 1,355 participants from the Korea

National Health and Nutrition Examination Survey (2014-2015). Logistic regression was used to analyze the associations across groups of MVPA-MSA guideline adherence (meet neither [reference]; MVPA only; MSA only; meet both MVPS-MSA) with MetS components (e.g., abdominal obesity, hypertriglyceridemia, low high-density lipoprotein cholesterol (HDL-C), hypertension, and hyperglycemia). The odds ratios (OR) were adjusted for covariates (e.g., sex, age, body mass index [BMI], and accelerometer wearing time). RESULTS: MSA only significantly reduced the OR for abdominal obesity (OR 0.34; 95\% confidence interval [CI] 0.13-0.91). When meeting both MVPA-MSA, the OR of having hypertriglyceridemia (OR 0.59; 95\% CI 0.39-0.88) and low HDL-C (OR 0.46; 95\% CI 0.310.68 ) reduced. Compared to meeting neither, MVPA only (OR 0.63; 95\% CI 0.44-0.89) and both MVPA-MSA (OR 0.46; 95\% CI 0.28-0.76) significantly reduced the OR for MetS. 
24 Korean adults do not meet both MVPA-MSA guidelines, public health actions to promote adherence should be supported.

KEY WORDS: Physical activity, Muscle-strengthening activity, Metabolic syndrome, Combined physical activity, Epidemiology

\section{INTRODUCTION}

Metabolic syndrome (MetS) is a collection of risk factors generally associated with cardiovascular disease and type-2 diabetes mellitus, which includes high blood pressure (BP), high cholesterol, elevated abdominal adiposity, and abnormal fasting plasma glucose $[1,2]$. Increased MetS risk factors are closely associated with mortality [3] and morbidity [4], as well as complications such as stroke, myocardial infarction, and angina [5]. The worldwide prevalence of MetS is estimated to be $20 \%-25 \%$ among adults [6, 7]. Similarly, the prevalence of MetS in Korea has steadily increased from $22.6 \%$ in 2013 to $30.4 \%$ in 2018 [8], and the high prevalence of MetS is considered a significant public health concern. The association between physical activity (PA) and metabolic health-related outcomes has been studied extensively [9-11]. For the prevention and treatment of MetS, the World Health Organization (WHO) recommends that adults should participate in aerobic moderate-tovigorous physical activity (MVPA) and follow the muscle-strengthening activity (MSA) guidelines (MVPA $\geq 150 \mathrm{~min} /$ week and MSA $\geq 2$ sessions/week) [12]. Given that these guidelines distinguish between the two types of activities (aerobic MVPA versus MSA), it may help in unique adaptations to particular physiological systems that improve the risk associated with a variety of MetS-related components. Despite the benefits of various PA reported on MetS, the epidemiological studies mostly discussed the benefits of MVPA [13, 14]. Over the last decade, emerging evidence on clinical exercise suggests that MSA can independently ameliorate or prevent MetS-related risk factors $[15,16]$. 
Although there are global recommendations on both types of activities (MVPA and MSA) and benefits of each type, there is a paucity of studies examining the association between combined MVPA-MSA and MetS [4]. Some studies indicated that meeting concurrent MVPA-MSA guidelines had the strongest association with MetS prevalence, as compared to meeting the individual guidelines for MVPA and MSA alone or meeting neither of them [17]. Among U.S. adults with hypertension $(n=155,791)$, high cholesterol $(n=141,173)$, and diabetes $(n=50,027)$, the lowest prevalence ratios (PRs) were seen in those who met combined MVPA-MSA, followed by MSA only, MVPA only, and met neither of the two guidelines (reference group) [18]. Despite the finding that meeting both MVPA-MSA recommendations has the most positive effect on MetS-related risk factors, most studies have been conducted mainly on Caucasians [4]. Therefore, whether these beneficial results can be generalized globally remains unclear. Given the possibility that associations between cardiometabolic risk factors may be dissimilar among ethnicities [19, 20], it is necessary to examine these associations in other ethnic groups, such as Asians. Moreover, although accelerometer-assessed MVPA objectively reported a stronger association with the MetS prevalence than self-reported MVPA [11, 21, 22], no study has confirmed the association between MetS and meeting both MVPA guidelines, which is measured by accelerometer and self-reported MSA recommendations simultaneously in Koreans.

Therefore, this study aimed to investigate whether meeting the combined guidelines of accelerometer-assessed MVPA and self-reported MSA simultaneously are associated with reduced odds of MetS than meeting neither or one of the guidelines among Koreans.

\section{MATERIALS AND METHODS}

\section{Participants}


73 The data for the study were collected from the 2014-2015 Korea National Health and

74 Nutrition Examination Survey (KNHANES), a nationally representative cross-sectional survey of samples from Korea that was conducted by the Korea Centers for Disease Control and Prevention (KCDC). The KNHANES is designed to collect the health and nutritional status information and to monitor trends of health risk factors and the prevalence of major chronic diseases in Korea.

Among the 2014-2015 KNHANES participants, adults who agreed to wear an accelerometer were selected for this analysis $(n=1,827)$. The following participants were excluded from the analysis: those with loss of accelerometer $(n=7)$, missing or error in accelerometer data $(n=47)$, accelerometer error $(n=3)$, insufficient wearing time of accelerometer $(n=342)$, and insufficient data on MetS-related risk factors and the MSA questionnaire ( $n=73)$. Finally, a total of 1,355 participants were eligible for this study (Fig 1).

\section{Accelerometer-assessed physical activity}

The accelerometer used in the KNHANES was wGT3X+ (ActiGraph, Florida, USA). The reading on the accelerometer was noted every day for a week from midnight on the day after the examination, and instructed to be worn at all possible times, except during water-based activities, such as swimming, bathing, and sleeping [23]. To ensure the assessment of daily PA patterns, individuals wore the accelerometer for at least 10 hours per day for a minimum of 4 days, and they were included in the analysis regardless of weekdays or weekends [24]. To distinguish the accelerometer non-wearing time, a minimum period of 60 consecutive minutes of zero activity count was defined, with an allowance of 1-2 minutes of activity counts between 0 and 100 [25]. MVPA was defined as >2020 activity counts per minute [25]. According to the PA guidelines for Americans, meeting PA guidelines was defined as 
accelerometer-determined MVPA of 600 metabolic equivalent of task (MET)-min/week or more, and every minute of MVPA was used [12].

\section{Self-reported muscle-strengthening activities}

The KNHANES, based on the Global Physical Activity Questionnaire (GPAQ), which is a self-reported questionnaire to survey PA along with the number of days of MSA, was investigated. The question on MSA in the questionnaire was as follows: 'In the past week, on how many days did you do push-ups, sit-ups, pull-ups, dumbbells or barbells training designed to strengthen your muscles?" The responses can range from "not at all" to "more than 5 days" with a weekly frequency. According to the PA guidelines for Americans, meeting the MSA guidelines was defined as individuals participating for more than 2 days a week [12].

\section{Metabolic syndrome}

The presence of MetS was determined according to the criteria established by the National Cholesterol Education Program Adult Treatment Panel III (NCEP ATP III) [26]. However, waist circumference was determined according to the Asian standard criteria [27]. Participants meeting three or more of the following five criteria were classified as having MetS: (1) waist circumference $\geq 90 \mathrm{~cm}$ in men and $\geq 85 \mathrm{~cm}$ in women; (2) serum triglyceride level of $\geq$ $150 \mathrm{mg} / \mathrm{dL}$; (3) high-density lipoprotein cholesterol (HDL-C) level $<40 \mathrm{mg} / \mathrm{dL}$ in men and < $50 \mathrm{mg} / \mathrm{dL}$ in women; (4) fasting glucose level $\geq 100 \mathrm{mg} / \mathrm{dL}$; (5) systolic $\mathrm{BP} \geq 130 \mathrm{mmHg}$ and/or diastolic $\mathrm{BP} \geq 85 \mathrm{mmHg}$. Medication users of dyslipidemia, diabetes, and hypertension were excluded from the study. 
122 In the present study, sex, age, family income, education, alcohol consumption, smoking, body 123 mass index (BMI), and accelerometer wearing time were selected as covariates [28-30]. Age

124 was used as a continuous variable and BMI was classified into 3 groups (underweight $<18.5$ $125 \mathrm{~kg} / \mathrm{m}^{2}$; normal $18.5 \mathrm{~kg} / \mathrm{m}^{2}-24.9 \mathrm{~kg} / \mathrm{m}^{2}$; and obese $\geq 25 \mathrm{~kg} / \mathrm{m}^{2}$ ) according to the Asian-Pacific 126 recommendations). Family income was divided into quartiles. Educational level was divided 127 into elementary school degree or less, middle school degree or less, high school degree or less, 128 and college or higher. Alcohol consumption was classified into three categories based on 129 respondents' reported alcohol consumption: a person who never drinks, a person who consumed 130 one drink or less per month in the past 1 year, and a person who consumed one drink or more 131 in the past 1 year. Smoking was divided into past smoking, non-smoking, and current smoking.

\section{Statistical analysis}

134 Baseline characteristics and adherence rate of PA guidelines according to MetS are presented 135 as mean (standard error) for continuous variables and as count and percentage for categorical 136 variables. A chi-square frequency test was used to assess the adherence rate of the PA 137 guidelines concerning MetS. Odds ratios (OR) and 95\% confidence intervals (CIs) were 138 obtained using logistic regression to analyze the associations between meeting only MVPA 139 guidelines, meeting only MSA guidelines, or meeting both MVPA and MSA guidelines with 140 MetS. Accelerometer raw data processing was performed using the SAS ver. 9.4 (SAS Institute, 141 Cary, NC, USA), and STATA (SE) 12.0 (Stata Corp., College Station, TX) was used for 142 statistical analysis. The statistical significance level was established as p-value of $<0.05$.

\section{Ethics statement}

145 This study protocol was approved by the Institutional Review Board of Seoul National 146 University (IRB no. E2011/001-011). Informed consent was obtained from each participant 147 during the survey. 
149

150

151

152

153

154

155

156

157

158

160

161

162

163

164

165

166

167

168

169

170

171

172

173

\section{RESULTS}

The baseline characteristics of the participants are presented in Table 1. The average age of 1,355 adults was 44.7 years, and $63.6 \%$ were females. Among the participants, $64.3 \%$ had normal BMI, 31.5\% were obese, and $4.1 \%$ were underweight. In PA categories, $37.6 \%$ of the participants did not meet combined or isolated MVPA and MSA guidelines, $41.5 \%$ met only MVPA guidelines, $6.0 \%$ met only MSA guidelines, and $14.7 \%$ met both guidelines. Additionally, in terms of MetS components, $21.1 \%$ had abdominal obesity, $30.1 \%$ had hypertriglyceridemia, 36.7\% had low HDL-C, $27.9 \%$ had hypertension, and $27.3 \%$ had hyperglycemia.

The adherence rate of PA guidelines according to MetS are described in Table 2. The proportion of people who did not meet neither PA guidelines was higher in the group with MetS compared to the group without MetS (41.6\% vs 36.4\%). On the other hand, the proportion of people meeting both PA guidelines was lower in the group with MetS compared to the group without MetS (11.7\% vs 15.6\%). MSA only (with MetS 6.1\%; without MetS 6.0\%) and MVPA only (with MetS 40.3\%; without MetS 41.8\%) showed similar proportions according to MetS. There was no statistical differences in the adherence rate of PA guidelines concerning MetS.

The associations of PA categories (reference group = met neither guideline) with MetS components (abdominal obesity, hypertriglyceridemia, low HDL-C, hypertension, and hyperglycemia) are described in Table 3. Meeting MSA only significantly reduced the odds ratios (OR) of having abdominal obesity (OR 0.34, 95\% CI 0.13-0.91). In contrast, MVPA did not show any statistically significant association. Nevertheless, when meeting combined MVPA-MSA, the ORs of having hypertriglyceridemia and low HDL-C were reduced (OR 0.59, 95\% CI: $0.39-0.88$; OR $0.46,95 \%$ CI $0.31-0.68)$.

The associations of PA categories (reference $=$ meet neither guideline) with MetS are shown 
174 in Figure 2. Compared to meeting neither guideline, meeting MVPA and both guidelines 175 significantly reduced the OR of having MetS, respectively (OR 0.63, 95\% CI 0.44-0.89; OR

$1760.46,95 \%$ CI $0.28-0.76$ ). On the other hand, MSA did not show significant association (OR $177 \quad 0.81,95 \%$ CI $0.40-1.62)$.

178

179 Discussion

180 We investigated for the first time the associations between different combinations of 181 accelerometer-measured MVPA and self-reported MSA guideline compliance with detrimental 182 MetS in a large sample of Asian adults. The main finding was that achieving the combined 183 MVPA-MSA guidelines was associated with independently more beneficial MetS-related outcomes than meeting neither, MVPA or MSA alone. Our key findings show that meeting both MVPA-MSA guidelines is independently associated with optimal MetS prevalence ratio in

186 Korean adults.

187 Currently, the epidemiological evidence on the association between PA and metabolic health

188 is based on aerobic MVPA guidelines. Both self-reported MVPA and objectively measured MVPA have reported many positive effects on MetS-related outcomes [31-34]. However, some studies have reported significant low-to-moderate correlations between objectively measured MVPA and self-reported MVPA [35, 36]. In particular, objectively measured MVPA using an accelerometer showed much stronger associations with MetS-related components than selfreported MVPA, even after adjusting for several potential confounders [11, 22]. Our results also showed that meeting the MVPA only significantly reduced the prevalence of MetS (OR $0.63,95 \%$ CI $0.44-0.89$ ). This is similar to the results of previous studies on populations in Western countries (OR 0.39, 95\% CI 0.31-0.48) [17]. However, the relationship between

197 MVPA only and MetS-related components was not statistically significant. These results may

198 be affected by whether the physical activity was measured objectively or subjectively, 
199 statistical techniques, and the differences in study participants (e.g., medical history of 200 participants and ethnicity). On the other hand, compared with studies on the epidemiology of 201 aerobic MVPA for decades, studies on MSA are scarce. Studies on clinical exercise show that 202 MSA is associated with metabolic health indicators (e.g., glucose/lipid metabolism, obesity, 203 and BP) [37, 38], skeletal muscle (e.g., bone density and hypertrophy) [39, 40], and functional 204 capacity (e.g., balance and performance) [41, 42]. However, we showed that meeting the MSA recommendation alone ( $\geq 2$ days/week) was not associated with MetS-related components other than waist circumference, which supports the findings of another study involving Korean adults [4]. These findings might be explained by the optimal dose, intensity, and frequency of MSA on metabolic health, which remain unclear, and bias due to subjective reporting (e.g., error in recall and overestimation of exercise) that cannot be excluded. In addition, since MSA requires relatively more exercise equipment (dumbbell and exercise machine), exercise skill

211 proficiency (e.g., to perform squats, push-ups, and deadlift), and understanding of terminology

212 and principles related to resistance exercises (e.g., set, repeated maximum, progression, and

213 overload), it is judged that it will be more difficult to achieve the recommendations for MSA

214 as compared to aerobic MVPA.

215 Our results provide early insights into the potentially beneficial role of MVPA, MSA, and combined MVPA-MSA on detrimental MetS-related components in a large population-based

217 sample. Our key findings support the extensive literature on the benefits of achieving both 218 aerobic and resistance PA recommendations for metabolic health. In particular, although there 219 was no decrease in the abdominal obesity, this reduction in MetS prevalence appears to have been achieved through the improvement of dyslipidemia. Furthermore, compared with previous studies in other countries, which predominantly included Caucasians as participants, we identified comparable beneficial MetS-related components for meeting combined MVPAMSA recommendations [17]. Our findings support the findings of Bennie et al. [4], who 
measured MVPA subjectively in Korean adults, and a longitudinal study showed that achieving both MVPA-MSA recommendations is associated with lower metabolic-related prevalence and components compared with achieving one recommendation [43]. These positive physiological effects may be explained by the fact that the combined effects of both MVPA and MSA can be greater than their individual effects [44].

In our study, only $14.7 \%$ of Korean adults met the combined MVPA-MSA recommendations. This achievement rate was similar to that in Australia (15\%) [45], but lower than that in Germany (22.6\%) [46] and the USA (20.2\%) [47]. Despite the growing number of potential health benefits of combined MVPA-MSA, approximately $80 \%-85 \%$ of currently reported sample populations from multiple countries do not meet the recommended aerobic MVPA and MSA guidelines from a public health perspective. Moreover, as a result of examining the adherence rate of PA guidelines according to the presence or absence of MetS in our study, the MVPA only and MSA only categories showed similar PA achievement rates whether with or without MetS. On the other hand, despite the many benefits of combined MVPA-MSA, people with MetS have a lower achievement rate with combined MVPA-MSA recommendations than those without MetS. Therefore, we need to closely examine the requirements for achieving both MVPA and MSA recommendations and support various strategies to promote adherence to both MVPA-MSA modes.

Our study has certain limitations. First, because the nature of cross-sectional studies makes it difficult to infer causality, the results of this study should be interpreted with caution. Future longitudinal studies that meet both MVPA and MSA guidelines are needed to clarify the associations between achieving both MVPA-MSA recommendations and MetS-related components. Second, our results were derived from subjects who measured PA using an accelerometer, which may have affected the results. For example, those excluded from the analysis for not wearing an accelerometer may be among the most physically inactive 
249 participants, which may have affected the results in less predictable ways. Lastly, MSA was

250 measured using self-reported questionnaires. Although the validity of this questionnaire was

251 proven, it may have been influenced by bias (e.g., over-estimated PA dose and recall bias). In

252 addition, the MSA questionnaire lacks in some amount of information about the frequency,

253 intensity, and time of participation compared to the MVPA questionnaire which may be

254 required for a more detailed analysis.

255

256 CONCLUSION

257 Among a sample of 1,355 Korean adults, combined accelerometer-measured aerobic MVPA

258 and self-reported MSA recommendation adherence was most beneficially associated with

259 MetS compared to meeting the MVPA and MSA recommendations only. Longitudinal

260 studies are needed to confirm the temporal relationship between meeting the MVPA-MSA

261 recommendations and metabolic health. Considering that more than $85 \%$ of Korean adults

262 cannot achieve both MVPA-MSA guidelines, public health actions to promote adherence

263 should be supported.

264

265

266 
268 1.Kassi E, Pervanidou P, Kaltsas G, Chrousos G. Metabolic syndrome: definitions and controversies. BMC medicine 2011;9:1-13.

2.Park SI, Suh J, Lee HS, Song K, Choi Y, Oh JS, et al. Ten-Year Trends of Metabolic Syndrome Prevalence and Nutrient Intake among Korean Children and Adolescents: A Population-Based Study. Yonsei medical journal 2021;62:344.

273

274 3.Bruno G, Merletti F, Biggeri A, Bargero G, Ferrero S, Runzo C, et al. Metabolic syndrome as a predictor of all-cause and cardiovascular mortality in type 2 diabetes: the Casale Monferrato Study. Diabetes care 2004;27:2689-2694.

4.Bennie JA, Ding D, Khan A, Stamatakis E, Biddle SJ, Kim J. Run, lift, or both? Associations between concurrent aerobic-muscle strengthening exercise with adverse cardiometabolic biomarkers among Korean adults. European journal of preventive cardiology 2020;27:738-748.

5.Moreira GC, Cipullo JP, Ciorlia LAS, Cesarino CB, Vilela-Martin JF. Prevalence of metabolic syndrome: association with risk factors and cardiovascular complications in an urban population. PloS one 2014;9:e105056. 6.Aschner P, Balkau B, Barter P, Bennett P, Boyko E, Brunzell J, et al. The IDF consensus worldwide definition of the Metabolic Syndrome. Available from: https://www.idf.org/elibrary/consensus-statements/60-idfconsensus-worldwide-definitionof-the-metabolicsyndrome.html.

7.do Vale Moreira NC, Hussain A, Bhowmik B, Mdala I, Siddiquee T, Fernandes VO, et al. Prevalence of Metabolic Syndrome by different definitions, and its association with type 2 diabetes, pre-diabetes, and cardiovascular disease risk in Brazil. Diabetes \& Metabolic Syndrome: Clinical Research \& Reviews 2020;14:1217-1224.

8.Statistics on the number of metabolic risk factors by age and gender [cited 2021. 07.07.]. Available from:

293 https://kosis.kr/statHtml/statHtml.do?orgId=350\&tblId=DT_35007_N136\&conn_path=I3.

294 9.Huang B, Hamer M, Chastin S, Pearson N, Koster A, Stamatakis E. Cross- sectional associations of device- measured sedentary behaviour and physical activity with cardiometabolic health in the 1970 British Cohort Study. Diabetic Medicine 2021;38:e14392. 10.Suliga E, Cieśla E, Rębak D, Kozieł D, Głuszek S. Relationship between sitting time, physical activity, and metabolic syndrome among adults depending on body mass index (BMI). Medical science monitor: international medical journal of experimental and clinical research 2018;24:7633.

11.Tucker JM, Welk GJ, Beyler NK, Kim Y. Associations between physical activity and metabolic syndrome: comparison between self-report and accelerometry. American Journal of Health Promotion 2016;30:155-162.

12.2018 Physical activity guidelines advisory committee scientific report. Available from: https://health.gov/our-work/physical-activity/current-guidelines/scientific-report.

13.Guthold R, Stevens GA, Riley LM, Bull FC. Worldwide trends in insufficient physical activity from 2001 to 2016: a pooled analysis of 358 population-based surveys with 1. 9 million participants. The lancet global health 2018;6:e1077-e1086. 14.Hallal PC, Andersen LB, Bull FC, Guthold R, Haskell W, Ekelund U, et al. Global physical activity levels: surveillance progress, pitfalls, and prospects. The lancet 2012;380:247-257.

15.Bennie JA, Lee D-c, Khan A, Wiesner GH, Bauman AE, Stamatakis E, et al. Musclestrengthening exercise among 397,423 US adults: prevalence, correlates, and associations with health conditions. American Journal of Preventive Medicine 2018;55:864-874. 
315 16.Churilla JR, Magyari PM, Ford ES, Fitzhugh EC, Johnson TM. Muscular strengthening

316 activity patterns and metabolic health risk among US adults. Journal of diabetes 2012;4:77-

31784

318 17.Dankel SJ, Loenneke JP, Loprinzi PD. The Individual, Joint, and Additive Interaction

319 Associations of Aerobic-Based Physical Activity and Muscle Strengthening Activities on

320 Metabolic Syndrome. International Journal of Behavioral Medicine 2016;23:707-713.

321 18.Bennie JA, De Cocker K, Teychenne MJ, Brown WJ, Biddle SJH. The epidemiology of aerobic physical activity and muscle-strengthening activity guideline adherence among 383,928 U.S. adults. International Journal of Behavioral Nutrition and Physical Activity 2019;16:34.

325 19.Kurian AK, Cardarelli KM. Racial and ethnic differences in cardiovascular disease risk

326 factors: a systematic review. Ethnicity and Disease 2007;17:143. 20.van Vliet M, von Rosenstiel IA, Schindhelm RK, Brandjes DPM, Beijnen JH, Diamant M. Ethnic differences in cardiometabolic risk profile in an overweight/obese paediatric cohort in the Netherlands: a cross-sectional study. Cardiovascular Diabetology 2009;8:2. 21.Sternfeld B, Gabriel KP, Jiang S-F, Whitaker KM, Jacobs Jr DR, Quesenberry Jr CP, et al. Risk estimates for diabetes and hypertension with different physical activity methods. Medicine and science in sports and exercise 2019;51:2498.

22.Celis-Morales CA, Perez-Bravo F, Ibañez L, Salas C, Bailey ME, Gill JM. Objective vs. self-reported physical activity and sedentary time: effects of measurement method on relationships with risk biomarkers. PloS one 2012;7:e36345.

336 23.KNHANES regulation for using of raw data 6th (2013-2015). Available from:

337 https://knhanes.kdca.go.kr/knhanes/sub03/sub03 06_02.do. 24.Troiano RP, Berrigan D, Dodd KW, Masse LC, Tilert T, McDowell M. Physical activity in the United States measured by accelerometer. Med Sci Sports Exerc 2008;40:181-188. 25.Troiano RP, Berrigan D, Dodd KW, Masse LC, Tilert T, McDowell M. Physical activity in the United States measured by accelerometer. Medicine and science in sports and exercise 2008;40:181.

26.Grundy SM, Cleeman JI, Daniels SR, Donato KA, Eckel RH, Franklin BA, et al. Diagnosis and management of the metabolic syndrome: an American Heart Association/National Heart, Lung, and Blood Institute scientific statement. Circulation 2005; 112:2735-2752. circumference cutoff points for central obesity in Korean adults. Diabetes research and clinical practice 2007;75:72-80. to Reduce Metabolic Syndrome Risk: A 10-Year Community-Based Prospective Study in Korea. Yonsei medical journal 2020;61:218-228.

29.Liu SH, Waring ME, Eaton CB, Lapane KL. Association of objectively measured physical activity and metabolic syndrome among US adults with osteoarthritis. Arthritis care \& research 2015;67:1371-1378.

356 30.Metzger JS, Catellier DJ, Evenson KR, Treuth MS, Rosamond WD, Siega-Riz AM. Associations between patterns of objectively measured physical activity and risk factors for the metabolic syndrome. American Journal of Health Promotion 2010;24:161-169. 31.Wu S, Fisher-Hoch SP, Reininger B, McCormick JB. Recommended levels of physical activity are associated with reduced risk of the metabolic syndrome in Mexican-Americans. PloS one 2016;11:e0152896.

32.He D, Xi B, Xue J, Huai P, Zhang M, Li J. Association between leisure time physical activity and metabolic syndrome: a meta-analysis of prospective cohort studies. Endocrine 2014;46:231-240. 
33.ATIENZA AA, MOSER RP, PERNA F, DODD K, BALLARD-BARBASH R, TROIANO RP, et al. Self-Reported and Objectively Measured Activity Related to Biomarkers Using NHANES. Medicine \& Science in Sports \& Exercise 2011;43:815-821. 34.Strasser B. Physical activity in obesity and metabolic syndrome. Annals of the New York Academy of Sciences 2013;1281:141-159.

35.Hagstromer M, Ainsworth BE, Oja P, Sjostrom M. Comparison of a subjective and an objective measure of physical activity in a population sample. Journal of Physical Activity and Health 2010;7:541-550.

36.Lim J, Sung H, Lee O, Kim Y. Physical activity in South Korea measured by accelerometer: The Korea National Health and Nutrition Examination Survey VI 2014-2015. Korean J. Sport Sci 2020;31:169-179.

37.Lemes ÍR, Ferreira PH, Linares SN, Machado AF, Pastre CM, Netto J. Resistance training reduces systolic blood pressure in metabolic syndrome: a systematic review and metaanalysis of randomised controlled trials. British Journal of Sports Medicine 2016;50:1438. 38.Artero EG, Lee D-c, Lavie CJ, España-Romero V, Sui X, Church TS, et al. Effects of Muscular Strength on Cardiovascular Risk Factors and Prognosis. Journal of Cardiopulmonary Rehabilitation and Prevention 2012;32

39.Schoenfeld BJ, Ogborn D, Krieger JW. Dose-response relationship between weekly resistance training volume and increases in muscle mass: A systematic review and metaanalysis. Journal of Sports Sciences 2017;35:1073-1082.

40.Martyn-St James M, Carroll S. Effects of different impact exercise modalities on bone mineral density in premenopausal women: a meta-analysis. Journal of bone and mineral metabolism 2010;28:251-267.

41.Mangione KK, Miller AH, Naughton IV. Cochrane review: Improving physical function and performance with progressive resistance strength training in older adults. Physical therapy 2010;90:1711-1715. 42.Steib S, Schoene D, Pfeifer K. Dose-response relationship of resistance training in older adults: a meta-analysis. Med Sci Sports Exerc 2010;42:902-914. 43.Stamatakis E, Lee I-M, Bennie J, Freeston J, Hamer M, O’Donovan G, et al. Does Strength-Promoting Exercise Confer Unique Health Benefits? A Pooled Analysis of Data on 11 Population Cohorts With All-Cause, Cancer, and Cardiovascular Mortality Endpoints. American Journal of Epidemiology 2017;187:1102-1112. 44.Dankel SJ, Loenneke JP, Loprinzi PD. Combined associations of muscle-strengthening activities and accelerometer-assessed physical activity on multimorbidity: findings from NHANES. American Journal of Health Promotion 2017;31:274-277. 45.Bennie JA, Pedisic Z, van Uffelen JGZ, Gale J, Banting LK, Vergeer I, et al. The descriptive epidemiology of total physical activity, muscle-strengthening exercises and sedentary behaviour among Australian adults - results from the National Nutrition and Physical Activity Survey. BMC Public Health 2016;16:73.

46.Bennie J, De Cocker K, Tittlbach S. Aerobic physical activity and muscle-strengthening exercise guideline adherence in Germany: findings from a national sample of 24,016 adults. 2020

47.Bennie JA, De Cocker K, Pavey T, Stamatakis E, Biddle SJ, Ding D. Muscle strengthening, aerobic exercise, and obesity: a pooled analysis of 1.7 million US adults. Obesity 2020;28:371-378. 
412 Figure 1. Flow diagram of study participant selection.

413

414 
415 Figure 2. Odds ratios (OR) with 95\% confidence intervals (95\% CI) for metabolic syndrome 416 among individuals meeting MSA and MVPA, and both guidelines. OR and 95\% CI are as 417 follows: MSA only: OR=0.81 (95\% CI 0.40-1.62), MVPA only: OR=0.63 (95\% CI 0.46418 0.89), Meet both: $\mathrm{OR}=0.46$ (95\% CI 0.28-0.76). The values were adjusted for the following 419 covariates: sex, age, family income, education, alcohol consumption, smoking, body mass 420 index (BMI), and accelerometer wearing time. 
Table_1. Characteristics of participants

\begin{tabular}{|c|c|c|}
\hline Variables & $\begin{array}{l}\text { Mean (standard error) } \\
\text { or number }(\%)\end{array}$ & 95\% Confidence interval \\
\hline Age (years) ${ }^{1}$ & $44.7(0.3)$ & $44.1-45.4$ \\
\hline Sex ( $\%$ female $)$ & $862(63.6)$ & $61.0-66.1$ \\
\hline \multicolumn{3}{|l|}{ Educational level (\%) } \\
\hline$<$ Elementary School & $112(8.2)$ & $6.7-9.7$ \\
\hline$<$ Middle School & $135(9.9)$ & $8.3-11.5$ \\
\hline$<$ High School & $545(40.2)$ & $37.6-42.8$ \\
\hline$>$ Undergraduate & $563(41.5)$ & $38.9-44.1$ \\
\hline \multicolumn{3}{|l|}{ Family income (\%) } \\
\hline$<25^{\text {th }}$ & $91(6.7)$ & $5.3-8.0$ \\
\hline $25-50^{\text {th }}$ & $343(25.3)$ & $22.9-27.6$ \\
\hline $50-75^{\text {th }}$ & 457 (33.7) & $31.2-36.2$ \\
\hline $75-100^{\text {th }}$ & $464(34.2)$ & $31.7-36.7$ \\
\hline \multicolumn{3}{|l|}{ Smoking $^{2}(\%)$} \\
\hline No & $1,175(86.7)$ & $84.9-88.5$ \\
\hline Yes & $180(13.2)$ & $11.4-15.0$ \\
\hline \multicolumn{3}{|l|}{$\operatorname{Alcohol}(\%)^{3}$} \\
\hline No & $612(45.1)$ & $42.5-47.8$ \\
\hline Yes & $743(54.8)$ & $52.1-57.4$ \\
\hline \multicolumn{3}{|l|}{ BMI (\%) } \\
\hline Underweight $\left(<18.5 \mathrm{~kg} / \mathrm{m}^{2}\right)$ & $56(4.1)$ & $3.0-5.1$ \\
\hline Normal $\left(18.5 \mathrm{~kg} / \mathrm{m}^{2}-24.9 \mathrm{~kg} / \mathrm{m}^{2}\right)$ & $872(64.3)$ & $61.8-66.9$ \\
\hline Obese $\left(\geq 25 \mathrm{~kg} / \mathrm{m}^{2}\right)$ & $427(31.5)$ & $29.0-33.9$ \\
\hline \multicolumn{3}{|c|}{ Physical activity guideline adherence (\%) } \\
\hline Meet neither & $510(37.6)$ & $35.0--40.2$ \\
\hline MVPA only ${ }^{4}$ & $563(41.5)$ & $38.9-44.1$ \\
\hline MSA only ${ }^{5}$ & $82(6.0)$ & $4.7-7.3$ \\
\hline Meet both & $200(14.7)$ & $12.8-16.6$ \\
\hline Metabolic syndrome (\%) & $307(22.6)$ & $20.4-24.8$ \\
\hline High waist circumference & $287(21.1)$ & $19.0-23.3$ \\
\hline High triglyceride & $408(30.1)$ & $27.6-32.5$ \\
\hline Low HDL-cholesterol & $498(36.7)$ & $34.1-39.3$ \\
\hline High blood pressure & $379(27.9)$ & $25.5-30.3$ \\
\hline High fasting glucose & $371(27.3)$ & $25.0-29.7$ \\
\hline
\end{tabular}

Data are presented as mean (standard error) $)^{1}$ or number (\%)

BMI: body mass index, MVPA: moderate-to-vigorous physical activity, MSA: muscle strengthening activity, HDL: highdensity lipoprotein

2Smoking: No means that 'have never smoked cigarette before' or 'past smoker,' Yes means that 'current smoker.'

${ }^{3}$ Alcohol: No means that 'have never consumed alcohol before' or 'less than one glass of alcohol was consumed in a month in a recent year,' Yes means that 'more than one glass of alcohol was consumed in a month in a recent year.'

${ }^{4}$ MVPA only: A group that meets the MVPA guidelines but not the MSA guidelines

${ }^{5}$ MSA only: A group that meets the MSA guidelines but not the MVPA guidelines 
Table 2. Adherence rate of physical activity guidelines according to metabolic syndrome

\begin{tabular}{cccc}
\hline & People with MetS & People without MetS & $\begin{array}{c}\text { Chi-Squared } \\
p \text {-value }\end{array}$ \\
\hline Meet neither & $128(41.6)$ & $382(36.4)$ & 0.095 \\
MSA only & $19(6.1)$ & $63(6.0)$ & 0.909 \\
MVPA only & $124(40.3)$ & $439(41.8)$ & 0.639 \\
Meet both & $36(11.7)$ & $164(15.6)$ & 0.088 \\
\hline
\end{tabular}

Data are presented as number (\%)

MetS: metabolic syndrome, MSA: muscle-strengthening activity, MVPA: moderate-to-vigorous physical activity 
Table 3. Adjusted odds ratios detailing the independent and combined effects of meeting MSA and MVPA guidelines

\begin{tabular}{|c|c|c|c|}
\hline MetS risk factors & Odds ratio & $95 \% \mathrm{CI}$ & $P$ value \\
\hline \multicolumn{4}{|l|}{ High waist circumference } \\
\hline Meet neither & reference & & \\
\hline MSA only & 0.34 & $0.13-0.91$ & 0.032 \\
\hline MVPA only & 0.92 & $0.58-1.45$ & 0.724 \\
\hline Meet both & 0.87 & $0.46-1.64$ & 0.673 \\
\hline \multicolumn{4}{|l|}{ High triglyceride } \\
\hline Meet neither & reference & & \\
\hline MSA only & 0.86 & $0.49-1.51$ & 0.613 \\
\hline MVPA only & 0.82 & $0.61-1.09$ & 0.179 \\
\hline Meet both & 0.59 & $0.93-0.88$ & 0.011 \\
\hline \multicolumn{4}{|l|}{ Low HDL-cholesterol } \\
\hline Meet neither & reference & & \\
\hline MSA only & 0.90 & $0.54-1.50$ & 0.691 \\
\hline MVPA only & 0.84 & $0.64-1.09$ & 0.191 \\
\hline Meet both & 0.46 & $0.31-0.68$ & $<0.001$ \\
\hline \multicolumn{4}{|l|}{ High blood pressure } \\
\hline Meet neither & reference & & \\
\hline MSA only & 1.41 & $0.79-2.25$ & 0.243 \\
\hline MVPA only & 1.27 & $0.94-1.71$ & 0.117 \\
\hline Meet both & 1.03 & $0.68-1.57$ & 0.859 \\
\hline \multicolumn{4}{|l|}{ High fasting glucose } \\
\hline \multicolumn{4}{|l|}{ Meet neither } \\
\hline MSA only & 1.07 & $0.59-1.93$ & 0.819 \\
\hline MVPA only & 1.03 & $0.76-1.39$ & 0.816 \\
\hline Meet both & 0.92 & $0.61-1.40$ & 0.712 \\
\hline
\end{tabular}

MetS: metabolic syndrome, CI: confidence interval, MSA: muscle-strengthening activity, MVPA: moderate-to-vigorous physical activity, HDL: high-density lipoprotein

Adjusted for the following covariates: sex, age, family income, education, alcohol consumption, smoking, body mass index (BMI), and accelerometer wearing time 


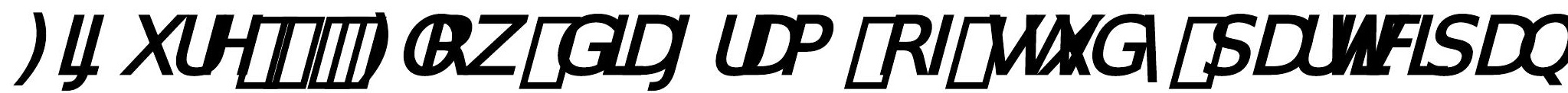

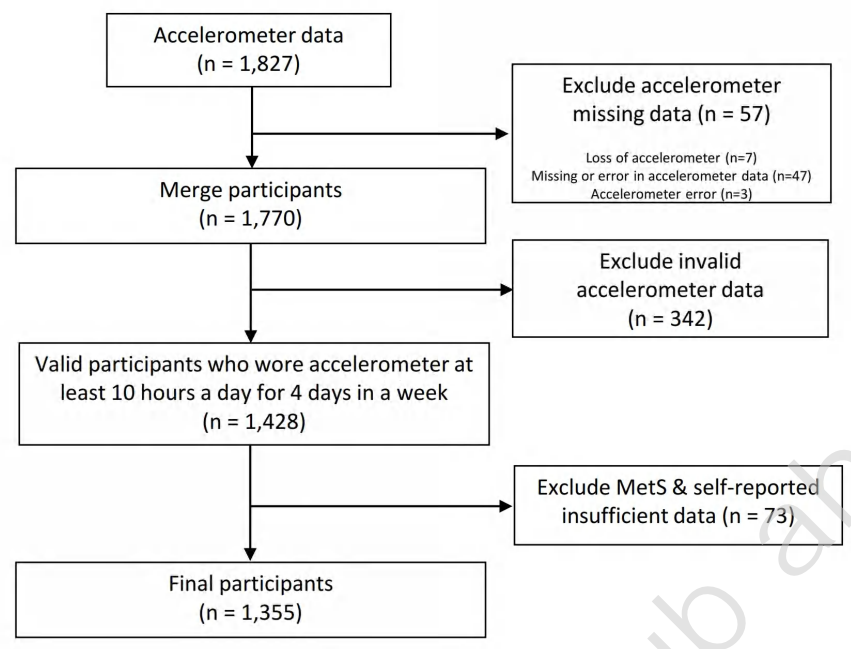




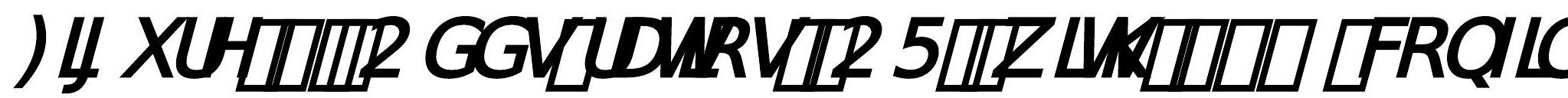

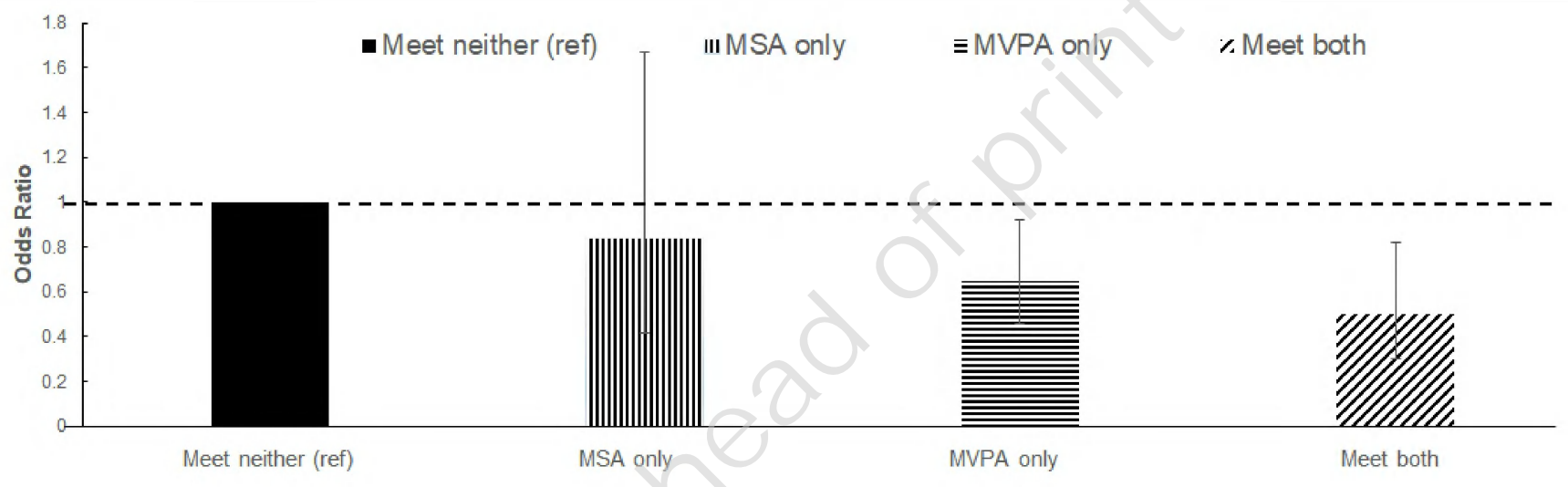

